Modern Physics Letters A

(C) World Scientific Publishing Company

\title{
Bohmian mechanics of Klein-Gordon equation via quantum metric and mass
}

\author{
S. Jalalzadeh \\ Departamento de Física, Universidade Federal de Pernambuco, \\ Recife, PE, 52171-900, Brazil \\ shahram@df.ufpe.br \\ A. J. S. Capistrano \\ Federal University of Latin-American Integration, \\ Foz do Iguaçu, Paraná 85867-970, Brazil \\ Casimiro Montenegro Filho Astronomy Center, Itaipu Technological Park, \\ Foz do Iguaçu, Paraná 85867-970, Brazil \\ abraao.capistrano@unila.edu.br
}

\begin{abstract}
The causal stochastic interpretation of relativistic quantum mechanics has the problems of superluminal velocities, motion backward in time and the incorrect non-relativistic limit. In this paper, according to the original ideas of de Broglie, Bohm and Takabayasi, we have introduced simultaneous quantum mass and quantum metric of curved spacetime to obtain a correct relativistic theory free of mentioned problems.

Keywords: Bohmian mechanics; Klein-Gordon equation; quantum conformal transformations.

PACS Nos.: 03.65.Ta, 03.65.Ca, 03.65.Pm
\end{abstract}

\section{Introduction}

There are various objections to use Klein-Gordon (KG) equation as a particle equation. It is well known the time component of current is not positive definite. Also, the probability density interpretation of squared modulus of wave function is inconsistent, because its integral through spatial part of spacetime would not be conserved in time. The usual solution these problems, in flat background Lorentzian manifold, is a rejection of the first quantized version of the KG equation in favor of the second quantization formalism given by Pauli and Weisskopf.$^{1}$ In curved spacetime the situation is more complicated and quantum fields lose their interpretation as asymptotic particles. Another problem is that unless the manifold has a global timelike killing vector, there is no way to define vacuum state in a canonical way. Moreover, at the fundamental level, it is not clear at all why the probability interpretation of wave function in non-relativistic quantum mechanics is in agreement with experiments, but not in the KG equation. ${ }^{2]}$ On the other hand, as argued by many authors,$\frac{3}{3}$ it seems that the causal stochastic interpretation of quantum me- 
chanics (SIQM) may have a solution to mentioned problems of relativistic quantum mechanics. According to the de Broglie-Bohm (dBB) ${ }^{4 / 5}$ in SIQM the wave function of quantum system describes a real field, called the quantum potential, which guides a particle along a trajectory having simultaneously well-defined position and velocity. The statistical aspects of quantum theory emerge through the uncontrollable character of both subquanta fluctuations and initial conditions. ${ }^{6}$ The novel feature of this interpretation is the appearance of the quantum potential, a highly nonlocal function of the parameters of particles and their environments. In fact, in the causal stochastic interpretation, it is shown that the claim of the Copenhagen interpretation according to which we must give up the concepts of causality, continuity and the objective reality of individual quantum objects is false ${ }^{77}$ On the other hand, the current form of the dBB interpretation of quantum mechanics is not fully satisfactory. The KG relativistic wave function leads to superluminal velocities and motion backward in time ${ }^{8}$ The aim of the present letter is to show that in the SIQM is possible to redefine the quantum mass and quantum metric of spacetime in which the superluminal velocities and backward motion in time will vanish.

\section{Causal stochastic interpretation of Klein-Gordon equation}

In this section, we assume the background spacetime $(\mathcal{M})$ equipped with $(g, \nabla)$ where $g$ is spacetime metric 2 -form with signature $(-,+,+,+)$ and $\nabla$ is the LeviCivita connection. Let $\Phi\left(x^{\mu}\right)$ be the KG wave function and $\mathcal{A}$ be the external electromagnetic 1-form such as $\mathcal{A}=A_{\mu} d x^{\mu} \in T^{*} \mathcal{M}$, where $T^{*} \mathcal{M}$ denotes the dual tangent space. Then the KG equation for a single particle with mass $m$ and electric charge $e$ will be

$$
\left[g^{\mu \nu}\left(i \nabla_{\mu}+e A_{\mu}\right)\left(i \nabla_{\nu}+e A_{\nu}\right)+m^{2}\right] \Phi\left(x^{\alpha}\right)=0 .
$$

Also, the conserved 4-current, corresponding to the KG equation (1) is

$$
J_{\mu}=\frac{e}{2 i m}\left(\Phi^{*} \nabla_{\mu} \Phi-\Phi \nabla_{\mu} \Phi^{*}\right)-\frac{e^{2}}{m} A_{\mu} \Phi \Phi^{*} .
$$

The KG equation (11) is separable by means of the general complex statement

$$
\Phi\left(x^{\mu}\right)=R\left(x^{\mu}\right) e^{i S\left(x^{\mu}\right)},
$$

where $R$ and $S$ are real functions of spacetime coordinates $x^{\mu}(\mu, \nu=0,1,2,3)$. Substituting (3) into the KG equation (11) gives ${ }^{9}$ 11

$$
g^{\mu \nu}\left(\nabla_{\mu} S-e A_{\mu}\right)\left(\nabla_{\nu} S-e A_{\nu}\right)+m^{2}\left(1-\frac{\square R}{m^{2} R}\right)=0,
$$

as the real part, and the conserved 4-current

$$
\nabla_{\mu} J^{\mu}=0
$$

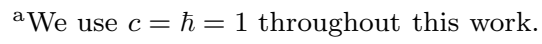


as the imaginary part, where $\square=g^{\alpha \beta} \nabla_{\alpha} \nabla_{\beta}=\frac{1}{\sqrt{-g}} \partial_{\mu}\left(\sqrt{-g} g^{\mu \nu} \partial_{\nu}\right)$ is the D' Alambert operator. In addition, the 4 -current $J_{\mu}$ can be reduced to

$$
J_{\mu}=\frac{e}{m} R^{2}\left(\nabla_{\mu} S-e A_{\mu}\right) .
$$

It is worth noting that equation (4) looks like the relativistic Hamilton-Jacobi equation (HJ), modified by dimensionless quantum potential $Q$ such that

$$
Q=-\frac{1}{m^{2}} \frac{\square R}{R} .
$$

The assumption introduced by the causal approach is that, in an analogous manner to the non-relativistic de Broglie-Bohm quantum mechanics, together with a location $x^{\mu}$ a particle has a well-defined 4-momentum

$$
p^{\mu}:=\nabla^{\mu} S=m\left(\frac{d x^{\mu}}{d \tau}+e A^{\mu}\right),
$$

as a set of equations with the aid of which momentum field, or equivalently 4 -velocity field

$$
u^{\mu}=\frac{d x^{\mu}}{d \tau}=\frac{1}{m}\left(\nabla^{\mu} S-e A^{\mu}\right),
$$

where $d \tau^{2}=-g_{\mu \nu} d x^{\mu} d x^{\nu}$ denotes the proper time. Inserting (8) into the (5) gives $\nabla_{\mu}\left(\rho u^{\mu}\right)=0$, which is the correct continuity equation for probability density (the background fluid density) $\rho=R^{2}$. From the modified HJ equation (4), it is clear that the identity $g_{\mu \nu} u^{\mu} u^{\nu}=-1$ is violated if the law of motion (8) and (9) can be applied, except in those special cases in which quantum potential vanish 9 [10 Moreover, de Broglie ${ }^{9}$ and Takabayas $\frac{10}{10}$ pointed out that this discrepancy might be corrected, and the law of motion can be maintained by attributing to a particle a quantum mass $M=m \sqrt{1+Q}$. On the other hand, as pointed out by Takabayasi,10 if we use the mentioned quantum mass in equations (5), (6) and (8) we find $\nabla_{\mu}\left(M R^{2} u^{\mu}\right)=0$, then it is no longer possible to consider the imaginary part of KG equation (5) as a continuity equation for background fluid density $\rho$, e.g. $\nabla_{\mu}\left(\rho u^{\mu}\right)=0$. Also, the 4 -velocity $u^{\mu}$ cannot, in general, have the meaning of a velocity vector field, because the variable mass $M$ can be imaginary (tachyonic) and the particle trajectory which start as a timelike trajectory becomes spacelike and can even move backwards in time. An alternative suggestion to solve the incompatibility of the law of motion with quantum HJ equation and unity of 4 -velocity, is to define a new metric for spacetime which is related conformally to the $g_{\mu \nu}$

$$
\tilde{g}_{\mu \nu}=(1+Q) g_{\mu \nu} .
$$

Then the HJ equation (4) will turn to

$$
\tilde{g}^{\alpha \beta}\left(\tilde{\nabla}_{\mu} S-e A_{\mu}\right)\left(\tilde{\nabla}_{\nu} S-e A_{\nu}\right)+m^{2}=0,
$$

where $\tilde{\nabla}$ denotes the Levi-Civita connection of $\tilde{g}$. Inserting $\tilde{\nabla}^{\mu} S-e A^{\mu}=m \frac{d x^{\mu}}{d \tau}$ into the (11) we obtain $\tilde{g}_{\alpha \beta} u^{\alpha} u^{\beta}=-1$. But this is not the ultimate remedy of 
the problem. The expectation is that the continuity equation (5), by inserting the above deformed metric and using (6), turn to the continuity equation for fluid as $\tilde{\nabla}_{\mu}\left(\rho u^{\mu}\right)=0$. It is easy to see that this is incompatible with the imaginary part of KG wave equation (5).

The last step, deformation of spacetime metric via quantum potential, sounds interesting in development of relativistic causal stochastic interpretation of quantum mechanics. As we know, the trajectory of particle is a hidden variable so as to be able to give a casual interpretation ${ }^{112}$ Hence, it seems that the metric structure of spacetime may be a hidden variable nature too. On the other hand, hidden variable nature of trajectories is in conflict with localized mass point of view of particles, i.e. the quantum effects eliminates point-like structures in favor of smeared objects. 13 Therefore, it may be natural to assume that both of particle mass and also the metric of spacetime are dependent to the quantum potential, or precisely

$$
\begin{aligned}
M & :=(1+Q)^{A} m, \\
\hat{g}_{\mu \nu} & :=(1+Q)^{B} g_{\mu \nu},
\end{aligned}
$$

where $A$ and $B$ are two dimensionless constants and $\hat{\nabla}$ denotes the covariant derivative respect to the deformed metric defined in equation (12). Now if we define the 4 -momentum as

$$
\begin{gathered}
p_{\mu}:=\hat{\nabla}_{\mu} S, \\
\hat{\nabla}_{\mu} S-e A_{\mu}=M u_{\mu},
\end{gathered}
$$

where $u^{\mu}=\frac{d x^{\mu}}{d \tau}$ is the 4 -velocity of particle in quantum spacetime $(\hat{\mathcal{M}}, \hat{g}, \hat{\nabla})$ and the proper time is given by $d \tau^{2}=-\hat{g}_{\mu \nu} d x^{\mu} d x^{\nu}$. Then, the consistency of quantum HJ equation (4) and $\tilde{g}_{\mu \nu} u^{\mu} u^{\nu}=-1$ gives $2 A+B=1$. Also the continuity equation (5) is equivalence to $\nabla_{\mu}\left(M \rho u^{\mu}\right)=(\sqrt{-g} M \rho)_{, \mu}=0$. To obtain the correct continuity equation for background fluid with density $\rho$, i.e., $\hat{\nabla}_{\mu}\left(\rho u^{\mu}\right)=\left(\rho \sqrt{-\hat{g}} u^{\mu}\right)_{, \mu}=0$, it is enough to put $M \sqrt{-g}=m \sqrt{-\hat{g}}$, which gives the second relation $2 B=A$. Hence we obtain

$$
\begin{aligned}
M & =(1+Q)^{\frac{2}{5}} m, \\
\hat{g}_{\mu \nu} & =(1+Q)^{\frac{1}{5}} g_{\mu \nu} .
\end{aligned}
$$

Inserting equations (13) and (14) into the (44) and (5) give the quantum HJ and continuity equations, respectively

$$
\begin{gathered}
\hat{g}^{\alpha \beta}\left(p_{\alpha}-e A_{\alpha}\right)\left(p_{\beta}-e A_{\beta}\right)+M^{2}=0, \\
\hat{\nabla}_{\mu}\left(\rho u^{\mu}\right)=0 .
\end{gathered}
$$

As we see from equation (14), the quantum mass $M$ is real for any value of quantum potential and consequently the theory is ghost free and the trajectory of particles remain timelike. The wave function (3) is invariant with respect to a change of its 
phase $S\left(x^{\mu}\right)$ by an integer multiple of $2 \pi \hbar$. Consequently definition of momentum (13) gives

$$
\oint p_{\mu} d x^{\mu}=\oint\left(M u_{\mu}+e A_{\mu}\right) d x^{\mu}=n h, \quad n=0,1,2, \ldots,
$$

as a condition of compatibility between equations (15) and (16) and KG equation (11). The path of integration may not pass through points or regions where probability density is zero because in this kinds of regions according to the equation of continuity (16) the velocity field is possibly singular. To obtain corresponding geodesic equation of quantum particle, we differentiate the 4-momentum defined in (13) with respect to the affine parameter of the trajectory, $d \tau$, which gives $\frac{d p_{\mu}}{d \tau}=u^{\alpha} \partial_{\alpha} \partial \mu S=u^{\alpha} \partial_{\mu} \hat{\nabla}_{\alpha} S$. Now using (13) and (15) we find

$$
\frac{d u^{\mu}}{d \tau}+\hat{\Gamma}_{\alpha \beta}^{\mu} u^{\alpha} u^{\beta}=-\frac{\partial_{\alpha} M}{M}\left(\hat{g}^{\alpha \mu}+u^{\alpha} u^{\mu}\right)+\frac{e}{M} F_{\alpha}^{\mu} u^{\alpha},
$$

where $F_{\mu \nu}=\partial_{\mu} A_{\nu}-\partial_{\nu} A_{\mu}$ denotes the electromagnetic field strength tensor. Equations (15) and (16) are now regarded as an ensemble of particle worldlines which belongs to a single 4 -velocity potential $S\left(x^{\mu}\right)$ by the relation (13) and is determined by the classical relativistic dynamics assuming the modified metric and mass by the quantum potential that is in turn dependent upon the density distribution of the ensemble. If a particle traverses a region of space where the Lorentz force, $(e / M) F_{\nu}^{\mu} u^{\nu}$, vanishes, but the electromagnetic potentials do not. Then geodesic equation (18) explains the Aharonov-Bohm effect since the particle is at all times acted upon by the quantum force (first term on the r.h.s. of (18)) and also moves in deformed space via quantum metric $\hat{g}[14$ Note that for vanishing values of quantum conformal factor, $Q+1=1-\frac{\square R}{m^{2} R}$, in quantum metric (14), the quantum metric $\hat{g}$ will be singular. Therefore, the quantum conformal boundary of spacetime $(\hat{\mathcal{M}}, \hat{g}, \hat{\nabla})$ is given by $\frac{\square R}{m^{2} R}=1$. Equivalently, the quantum conformal boundary of spacetime, $\partial \hat{\mathcal{M}}$ is given by those regions of spacetime that $R$ fluctuates greatly of the order of the Compton wave length of particle, $1 / \mathrm{m}$. Therefore, the observer does not have any access to the region outside.

In the non-relativistic approximation, $\left(u^{0} \approx 1,\left|d x^{i} / d t\right| \ll 1, \tilde{g}_{00} \approx-1, i=\right.$ $1,2,3)$, the conformal factor reduces to

$$
Q=-\frac{1}{m^{2}} \frac{\square R}{R} \approx-\frac{1}{m^{2}} \frac{\partial_{i} \partial^{i} R}{R}=\frac{2}{m} U,
$$

where $U=-\frac{1}{2 m} \partial_{i} \partial^{i} R / R$ is the non-relativistic quantum potential ${ }^{[5}$ Consequently one can find that the geodesic equation (18) reduces to the Euler equation of the Madelung fluid equation

$$
\frac{d^{2} x^{i}}{d t^{2}}=-\partial^{i}(\phi+U)+\frac{e}{m}\left(E^{i}+\epsilon_{i j k} B^{k} \frac{d x^{j}}{d t}\right),
$$

where $\phi$ is the weak gravitational potential, $E^{i}$ and $B^{i}$ are the components of electric and magnetic fields, respectively. As expected, we obtain the correct non- 
relativistic limit ${ }^{5}$ and the particle feels the usual Lorentz, weak gravitational field plus the non-relativistic quantum forces.

\section{Conclusion}

It was shown that with simultaneous quantum conformal transformations of mass of relativistic particles and the metric of spacetime, it is possible to write a covariant casual stochastic quantum equations of motion for a relativistic particle with no problems of superluminal velocities and the incorrect non-relativistic limit. This shows that the inertia of a quantum particle and metric structure of spacetime are determined by a scalar field which is a function of all relevant quantum processes occurring in the environment of the particle. Therefore, the quantum potential plays the role of the scalar field introduced by Brance and Dicke to supplement Einstein's general theory of relativity with an explicit statement of Mach's principle.

\section{References}

1. W. Pauli and V.F. Weisskopf, Helv. Phys. Acta 1709 (1934).

2. H. Nikolic, Found. Phys. Lett. 18549 (2005).

3. H. Nikolic, Int. J. Mod. Phys. A 226243 (2007); A Shojai and F. Shojai, Physica Scripta, 64413 (2001); N. Cufaro-Petroni, C. Dewdney, P. Holland, T. Kyprianidis, and J. P. Vigier, Phys. Lett. A 106368 (1984); C. Dewdney, P. R. Holland, A. Kyprianidis, and J. P. Vigier, Nature 336536 (1988); A. Kyprianidis, Phys. Lett. A 111, 111 (1985); P. R. Holland and J. P. Vigier, Nuovo Cimento 88B 20 (1985); G. Horton, C. Dewdney, and A. Nesteruk, J. Phys. A 337337 (2000); S. Hernandez-Zapata and E. Hernandez-Zapata, Found. Phys. 40, 532 (2010); H. Nikolic, Found. Phys. 42632 (2012); F. Shojai and M. Golshani, Int. J. Mod. Phys. A 13, 677 (1998); F. Shojai, A. Shojai and M. Golshani, Mod. Phys. Lett. A 132725 (1998); F. Shojai, A. Shojai and M. Golshani, Mod. Phys. Lett. A 132915 (1998).

4. L. de Broglie, Non-Linear Wave Mechanics (Elsevier, Amsterdam, 1960).

5. D. Bohm, Phys. Rev., 85 (1952) 166; D. Bohm, Phys. Rev., 85180 (1952); D. Bohm and B.J. Hiley, The undivided universe (Routledge, 1993).

6. M. Cini, Nuovo Cimento B, 73 (1983) 27.

7. D. Dürr, S. Goldstein and N. Zanghi, Quantum Physics Without Quantum Philosophy (New York: Springer, 2012); P.R. Holland The Quantum Theory of Motion: An Account of the De Broglie-Bohm Causal Interpretation of Quantum Mechanics (Cambridge, UK: Cambridge Univ. Press 1993).

8. C. Dewdney, G. Horton, M. M. Lam, Z. Malik, and M. Schmidt, Found. Phys. 22, 1217 (1992); H. Nikolić, Found. Phys. Lett. 18, 549 (2005).

9. L. de Broglie, Journ. de Phys., 8, 225 (1927).

10. T. Takabayasi, Prog. Theor. Phys., 9, 187 (1953); T. Takabayasi, Prog. Theor. Phys., 8143 (1952).

11. K. F. Novobatzky, Ann. der Phys. (Leipzig), 6. Folge, 11, 285 (1953).

12. K. Berndl, M. Daumer, D. Dürr, S. Goldstein and N. Zanghi, Il Nuovo Cim. B 110, 737 (1995).

13. P. Nicolini, A. Smailagic and E. Spallucci, Phys. Lett. B 632, 547 (2006).

14. P.R. Holland, Ann. NY Acad. Sci. 480, 579 (1986); D. Dürr, S. Goldstein, J. Taylor, R. Tumulka and N. Zanghi, Annales Henri Poincare 7, 791 (2006); ] C.-C. Chou and R.E. Wyatt, Ann. Phys. 325, 2234 (2010). 
15. R. Marnelius, Acta Phys. Pol. B 13, 669 (1982).

16. J. D. Bjorken, Lecture Notes on Current-Induced Reactions, eds. J. Komer et al. (Springer, 1975). 